\title{
On the Selection Rules for Spin-Lorentz Cobordisms
}

\author{
Domenico Giulini * \\ Fakultät für Physik der Universität Freiburg, Hermann-Herder-Strasse 3, \\ W-7800 Freiburg i. Br., Federal Republic of Germany
}

Received December 17, 1991

\begin{abstract}
A recent result of Gibbons and Hawking on the existence of spinLorentz cobordisms is applied to the class of 3-manifolds that can support a nonnegative scalar curvature metric. We call such manifolds admissible. It is shown how in general the existence of a spin-Lorentz cobordism restricts the numbermod- 2 of certain prime manifolds to occur in the prime decomposition, which are explicitly listed in the case of admissible manifolds.
\end{abstract}

\section{Introduction}

Very recently, Gibbons and Hawking derived a necessary and sufficient condition for a closed, orientable 3-manifold, $\Sigma$, to spin-Lorentz bound, that is, to be the spacelike boundary of a Lorentzian 4-Manifold, $M$, that admits a $S L(2, C)$-spin structure [2]. Their result was that the mod-2 Kervaire semi-characteristic, $\mathscr{U}(\Sigma)$, must be zero. Here, $\mathscr{U}(\Sigma)$ can itself be defined through the combination of cohomology groups:

$$
\begin{gathered}
\mathscr{S}(\Sigma)=\operatorname{dim} H^{0}\left(\Sigma, Z_{2}\right)+\operatorname{dim} H^{1}\left(\Sigma, Z_{2}\right), \\
\mathscr{U}(\Sigma)=\mathscr{S}(\Sigma) \bmod 2 .
\end{gathered}
$$

In Sect. 1 we want to reformulate this expression in terms of homology groups with integer coefficients and use it to derive a simple behaviour of $\mathscr{U}(\Sigma)$ under taking disjoint and connected sums. This is summarized in Corollary 1. In Sect. 2 we restrict attention to the so-called admissible 3-manifolds, which are characterized by admitting a metric of non-negative scalar curvature. Assuming a standard conjecture in 3-manifold theory to hold, we can give an explicit list of those prime manifolds whose occurrence is restricted $\bmod 2$ if their connected sum is required to spin-Lorentz bound, or if required to be spin-Lorentz cobordant to

^ e-mail: giulini@sun1.ruf.uni-freiburg.de 
Table 1

\begin{tabular}{lll}
\hline$\pi_{1}(\Sigma)$ & $H_{1}(\Sigma)$ & $\mathscr{U}(\Sigma)$ \\
\hline$Z_{p}$ & $Z_{p}$ & $\frac{1}{2}\left(1-(-1)^{p}\right)$ \\
$D_{8 n}^{*} \times Z_{q}$ & $Z_{2} \times Z_{2} \times Z_{q}$ & 1 \\
$D_{4(2 n+1)}^{*} \times Z_{q}$ & $Z_{4} \times Z_{q}$ & 0 \\
$T^{*} \times Z_{q}$ & $Z_{3} \times Z_{q}$ & 1 \\
$O^{*} \times Z_{q}$ & $Z_{2} \times Z_{q}$ & 0 \\
$I^{*} \times Z_{q}$ & $Z_{q}$ & 1 \\
$D_{2^{k} \cdot(2 n+1)}^{\prime} \times Z_{q}$ & $Z_{2^{k}} \times Z_{q}$ & 0 \\
$T_{8^{\prime} 3^{j}}^{\prime} \times Z_{q}$ & $Z_{3^{k}} \times Z_{q}$ & 1 \\
$Z_{1}$ & $Z$ & 0 \\
$G_{2}$ & $Z \times Z \times Z$ & 0 \\
$G_{3}$ & $Z \times Z_{2} \times Z_{2}$ & 0 \\
$G_{4}$ & $Z \times Z_{3}$ & 0 \\
$G_{5}$ & $Z \times Z_{2}$ & 1 \\
$G_{6}$ & $Z$ & 0 \\
\hline
\end{tabular}

$S^{3}$. This is summarized in Corollary 2 and Table 1. Although there is a way (not $1-1$ ) of counting 3-manifolds in which the non-admissible ones form most of all 3-manifolds (see e.g. [8]), they can be argued to be of less interest for applications in General Relativity $[1,8]$. In that sense Corollary 2 seems to cover the general relativist's demands so far.

\section{Behaviour under Disjoint and Connected Sums}

We start by noting that the first term in (1) simply counts the number, $n$, of connected components of $\Sigma$. Then, using standard theory of (co)homology (see e.g. [5]), one shows that for $\Sigma$ having the connected components $\left\{\Sigma_{1}, \ldots, \Sigma_{n}\right\}$

$$
H^{1}\left(\Sigma, Z_{2}\right) \cong \bigoplus_{i=1}^{n}\left\{F H_{1}\left(\Sigma_{i}, Z\right) \otimes Z_{2} \oplus \operatorname{Tor}\left(H_{1}\left(\Sigma_{i}, Z\right), Z_{2}\right)\right\},
$$

where Tor $\left(G, Z_{2}\right)$ simply denotes the kernel of the "squaring" map on the abelian group $G: G \rightarrow G ; g \mapsto 2 g$, and $F H_{1}$ denotes the free part of the finitely generated abelian group $H_{1}\left(\Sigma_{i}, Z\right)$ :

$$
H_{1}\left(\Sigma_{i}, Z\right)=\left(\bigoplus_{k=1}^{f_{i}} Z\right) \oplus\left(\bigoplus_{l=1}^{t_{i}} Z_{p_{l i}}\right)=: F H_{1}\left(\Sigma_{i}, Z\right) \oplus T H_{1}\left(\Sigma_{i}, Z\right) .
$$

The second bracket, containing the cyclic groups of finite order, is called the torsion part $\left(T H_{1}\right)$. Its representation as a direct sum of cyclic groups of order $p_{l i}$ is certainly not unique since $Z_{p} \oplus Z_{q} \cong Z_{p q}$ for $p, q$ any pair of coprime integers. Uniqueness can be achieved by the requirement that all $p_{l i}$ be powers of prime numbers. In any case, the number of even $p_{l i}$ 's is easily seen to be invariant. We will call it the even-torsion-rank $\tau_{i}$, and $f_{i}$ the free-rank. The latter one, usually just called the rank, is equal to the dimension of the first DeRahm cohomology group, that is, to the dimension of the space of harmonic 1 -forms on $\Sigma$. The significance of $\tau_{i}$ is that only a cyclic group of even order has non-trivial elements that square to 
the identity, so that one has

$$
\operatorname{Tor} H_{1}\left(\Sigma_{i}, Z_{2}\right) \cong \bigoplus_{l=1}^{\tau_{i}} Z_{2}
$$

Using (5) and (4) in (3) gives

$$
\mathscr{U}(\Sigma)=\left(\sum_{i=1}^{n} \mathscr{U}\left(\Sigma_{i}\right)\right) \bmod 2=\left(n+\sum_{i=1}^{n}\left(f_{i}+\tau_{i}\right)\right) \bmod 2 .
$$

Next, we assume that $\Sigma$ is connected and given by the connected sum of $n$ manifolds $\left\{\Sigma_{1}, \ldots, \Sigma_{n}\right\}$. It is well known (see e.g. [5]) that the fundamental group of this connected sum is given by the free product of the fundamental groups of the individual factors. The first homology group with integer coefficients, being just the abelianisation of the fundamental group, is therefore just the direct sum of the individual ones. If we again denote by $f_{i}$ and $\tau_{i}$ the free- and even-torsion-rank of the $\Sigma_{i}$ 's, we have

$$
\mathscr{U}(\Sigma)=\left(1+\sum_{i=1}^{n}\left(t_{i}+\tau_{i}\right)\right) \bmod 2=\left(1+\sum_{i=1}^{n}\left(1+\mathscr{U}\left(\Sigma_{i}\right)\right)\right) \bmod 2 .
$$

We call a manifold nuclear, if its characteristic $\mathscr{U}$ vanishes and non-nuclear otherwise. Note that a manifold is non-nuclear if and only if it is spin-Lorentzcobordant to the 3-sphere. We can now use Eqs. (6) and (7) to state a corollary to the theorem of Gibbons and Hawking.

Corollary 1. A) The disjoint sum of closed orientable 3-manifolds is nuclear (respectively non-nuclear), if and only if there is an even (respectively odd) number of non-nuclear connected components.

B) The connected sum of 3-manifolds is nuclear (respectively non-nuclear), if and only if the number of nuclear factors is odd (respectively even).

\section{Restriction to Admissible Manifolds}

From now on, we take the word manifold to also imply connected, closed and orientable. Since any manifold can be uniquely decomposed into a finite connected sum of so-called prime manifolds, we can calculate the characteristic $\mathscr{U}(\Sigma)$ once we know it for the prime factors.

A list of all prime manifolds is, however, not known and we shall therefore restrict attention to admissible prime manifolds, already characterized in the introduction. For this we look at the following three classes of prime 3-manifolds: The first class comprises all manifolds of finite fundamental group. Amongst those are the elliptic spaces, $S^{3} / G$, where $G$ is any finite subgroup of $S O(4)$ that acts freely via the action induced from the standard orthogonal action of $S O(4)$ on $S^{3}$. It is at present an undecided conjecture that any 3-manifold of finite fundamental group is homeomorphic to such an elliptic space. Besides the Poincaré conjecture, this involves a conjecture stating that the groups listed are all the freely acting ones, and that these can act only via the orthogonal action (up to conjugation by any homeomorphism). This is known to hold for most but not all of the groups. See [7] for more information on this. We shall now assume this conjecture to hold, that is, we assume that any manifold of finite fundamental group is homeomorphic to an elliptic space $S^{3} / G$. There is a countable infinity of 
such groups which have the conventional names: $Z_{p}, D_{4 m}^{*} \times Z_{q}, T^{*} \times Z_{q}, O^{*} \times Z_{q}$, $I^{*} \times Z_{q}, D_{2^{k} \cdot(2 n+1)}^{\prime} \times Z_{q}$, and $T_{8 \cdot 3_{j}}^{\prime} \times Z_{q}$, where the range of the integers $p, m, k, n, j$, $q$ is $m, k \geqq 2 ; p, k, j, q \geqq 1$, where $q$ has to be coprime to the order of the group the $Z_{q}$ is multiplied with. In particular, $q$ has to be odd. $D_{4 m}^{*}, T^{*}, O^{*}$, and $I^{*}$ denote $S U(2)$ double covers of the dihedral, tetrahedral, octahedral, and icosahedral groups of orders $4 m, 24,48$, and 120 , respectively. $D_{2^{k \cdot(2 n+1)}}^{\prime}$ and $T_{8 \cdot 3^{j}}^{\prime}$ can be understood as extensions of $Z_{2^{k-2}}$ and $Z_{3^{j-1}}$ by $D_{4(2 n+1)}^{*}$ and $T^{*}$, respectively. Presentations of these groups can be found in [6]. Together they comprise all 3-manifolds of constant, positive curvature.

The second class just contains the 3-handle, $S^{1} \times S^{2}$, and the third class contains all manifolds of the form $R^{3} / G$, where $G$ is a freely, properly discontinuously acting subgroup of the 3-dimensional Euclidean group, $E_{3}$, with compact, orientable quotient space. There are six such groups, which, in standard notation [9], we denote by $G_{1}, \ldots, G_{6}$. $G_{1}$ is just $Z \times Z \times Z$ with the 3-torus as resulting quotient manifold, and $G_{2}, G_{3}, G_{4}, G_{5}, G_{6}$ are certain extensions of the groups $Z_{2}, Z_{3}, Z_{4}$, $Z_{6}, Z_{2} \times Z_{2}$ by $G_{1}$, respectively. Presentations of these can be found in [9]. It follows that the latter five quotient manifolds are finitely covered by the 3-torus. Together they comprise all flat 3-manifolds.

It is known that a manifold admits a metric of everywhere positive scalar curvature if and only if its prime decomposition consists entirely of elliptic spaces and handles [3]. Moreover, any connected sum in which any other primemanifold appears and which allows for a non-negative scalar curvature metric must in fact be flat and therefore homeomorphic to one of the six manifolds $R^{3} / G_{1}, \ldots, R^{3} / G_{6}$. Up to the conjecture mentioned, the manifolds listed are therefore sufficient to generate (via connected sums) those manifolds that admit metrics with nowhere negative scalar curvature. These metrics are required in a variety of applications, like 1) for the existence of maximal spatial slicings of spacetimes [8], 2) for the transition (nucleation) from Euclidean to Lorentzian spacetimes [1], and 3) to ensure the absence of harmonic spinors [4].

Since the fundamental group of $S^{3} / G$ or $R^{3} / G$ is just $G$, we easily obtain the first homology by abelianisation and thus the characteristic $\mathscr{U}(\Sigma)$. The result is summarized in Table 1 . Here the manifolds are sufficiently characterized by their fundamental group, whereby $\pi(\Sigma)=Z$ corresponds to $\Sigma=S^{1} \times S^{2}$.

We now call the nuclear (respectively non-nuclear) elliptic spaces $\mathscr{N}_{E}$ (respectively $\overline{\mathscr{N}}_{E}$ ), the nuclear (respectively non-nuclear) flat spaces $\mathscr{N}_{F}$ (respectively $\overline{\mathscr{N}}_{F}$ ) and have, by inspection from Table 1 ,

$$
\begin{array}{r}
S^{3} / G \in \begin{cases}\mathscr{N}_{E}, & \Leftrightarrow G \in\left\{Z_{2 p}, D_{4(2 n+1)}^{*} \times Z_{q}, O^{*} \times Z_{q}, D_{2^{k} \cdot(2 n+1)}^{\prime} \times Z_{q}\right\} \\
\overline{\mathcal{N}}_{E}, & \Leftrightarrow G \in\left\{Z_{2 n+1}, D_{8 n}^{*} \times Z_{q}, T^{*} \times Z_{q}, I^{*} \times Z_{q}, T_{8 \cdot 3^{k}}^{\prime} \times Z_{q}\right\},\end{cases} \\
R^{3} / G \in \begin{cases}\mathscr{N}_{F}, & \Leftrightarrow G \in\left\{G_{1}, G_{2}, G_{3}, G_{5}\right\} \\
\overline{\mathcal{N}}_{F}, & \Leftrightarrow G \in\left\{G_{4}, G_{6}\right\} .\end{cases}
\end{array}
$$

Given the validity of the conjecture mentioned above, and making essential use of the theorem of Gromov and Lawson, we have

Corollary 2. An admissible manifold $\Sigma$ is nuclear (respectively non-nuclear), if and only if its prime decomposition contains either an odd (respectively even) number of manifolds from $\mathcal{N}_{E} \cup\left\{S^{1} \times S^{2}\right\}$, or is given by a single prime-manifold from $\mathscr{N}_{F}$ (respectively $\overline{\mathscr{N}}_{\boldsymbol{F}}$ ). 


\section{References}

1. Gibbons, G.W., Hartle, J.: Phys. Rev. D 42, 2458 (1990)

2. Gibbons, G.W., Hawking, S.W.: Selection rules for topology change. Commun. Math. Phys. 148, 345-352 (1992)

3. Gromov, M., Lawson, H.B.: I.H.E.S. Publicationes Mathematiques 58 (1983)

4. Lichnerowicz, A.: Comp. Rend. Acad. Sci. Paris, Sér. A-B 257, 7-9 (1963)

5. Maunder, C.R.F.: Algebraic topology. Cambridge: Cambridge University Press 1980

6. Orlik, P.: Seifert manifolds. Lecture Notes in Mathematics, Vol. 291. Berlin, Heidelberg, New York: Springer 1972

7. Thomas, C.B.: Elliptic structures on 3-manifolds. London Math. Soc. Lecture Note Series 103. Cambridge: Cambridge University Press (1986); also: Bull. London Math. Soc. 20, 65-67 (1988)

8. Witt, D.: Topological obstructions to maximal slices. UCSA Preprint, University of California, Santa Barbara (1987)

9. Wolf, J.A.: Spaces of constant curvature. Berkeley, CA: Publish or Perish 1977

Communicated by N. Yu. Reshetikhin 
